\title{
AUTOMATIC CONTINUITY OF CONCAVE FUNCTIONS
}

\author{
ROGER HOWE
}

(Communicated by William J. Davis)

\begin{abstract}
A necessary and sufficient condition is given that a semicontinuous, nonnegative, concave function on a finite dimensional closed convex set $X$ necessarily be continuous at a point $x_{0} \in X$. Application of this criterion at all points of $X$ yields a characterization, due to Gale, Klee and Rockafellar, of convex polyhedra in terms of continuity of their convex functions.
\end{abstract}

Let $V$ be a real vector space of dimension $n<\infty$. Let $X \subseteq V$ be a closed convex body. Let $\phi$ be a concave, nonnegative function on $X$. (Recall $\phi$ is concave if $-\phi$ is convex.) Define $G^{-}(\phi)$, the subgraph of $\phi$, as the subset of $V \times \mathbf{R}$ specified by

$$
G^{-}(\phi)=\{(x, t): x \in X, 0 \leq t \leq \phi(x)\} .
$$

If $\phi$ is not identically zero then $G^{-}(\phi)$ will be a convex body in $V \times \mathbf{R}$. We call $\phi$ semicontinuous if $G^{-}(\phi)$ is a closed subset of $V \times \mathbf{R}$. (This is usually called upper semicontinuity; since lower semicontinuity is not very important here, we let the "upper" be understood implicitly.) Observe that this is equivalent to the superlevel sets

$$
L^{+}(\phi, s)=\{x \in X: s \leq \phi(x)\}, \quad s \geq 0,
$$

being closed. Observe also that the $L^{+}(\phi, s)$ are convex.

We say $X$ is polyhedral if it is specified by a finite number of linear inequalities

$$
X=\left\{v \in V: \lambda_{i}(v) \leq b_{i}, \lambda_{i} \in V^{*}, b_{i} \in \mathbf{R}, 1 \leq i \leq m\right\} .
$$

In [GKR] (see also $[\mathbf{R}, \S 10]$ ) it is shown that if $\phi$ is a nonnegative, concave, semicontinuous function on $X$, and $X$ is polyhedral, then $\phi$ is in fact continuous. (Actually, in [GKR], convex functions are considered; but concave and convex are interchangeable here.) The purpose of this note is to refine the result by giving a pointwise criterion for automatic continuity. If our condition holds at all points of a convex set $X$, then $X$ is close to being polyhedral. (More precisely it is boundedly polyhedral in the sense of [GKR]; see Proposition 3.)

With $X$ as above, suppose that for some $t>0$ we have a closed convex set of $Y \subseteq V \times[0, t]$ such that

$$
\begin{aligned}
& \text { (a) } Y \cap(V \times\{0\})=X \times\{0\} \\
& \text { (b) If }(x, r) \in Y \text {, then }\left(x, r^{\prime}\right) \in Y \text { for } 0 \leq r^{\prime} \leq r .
\end{aligned}
$$

Received by the editors December 18, 1986 and, in revised form, May 4, 1987. 1980 Mathematics Subject (lassification (1985 Revision). Primary 52A20.

Key words and phrases. Concave function, semicontinuity, continuity. 
Then the recipe

(c) $\phi_{Y}(x)=\max \{r:(x, r) \in Y\}, x \in X$, defines a concave nonnegative function on $X$, and

(d) $Y=G^{-}\left(\phi_{Y}\right)$.

Denote by $\operatorname{SCNC}(X)$ the set of semicontinuous, nonnegative concave functions on $X$. It is straightforward to check that the sum of two functions in $\operatorname{SCNC}(X)$ is again in $\operatorname{SCNC}(X)$. Also a positive scalar multiple of an element in $\operatorname{SCNC}(X)$ is again an element. Thus $\operatorname{SCNC}(X)$ is a cone in the space of all real-valued functions on $X$. Also given a family $\left\{\phi_{i}\right\}_{i \in I}$ of functions in $\operatorname{SCNC}(X)$ (the index set $I$ may be infinite), we may form their infimum

$$
\inf \left\{\phi_{i}\right\}(x)=\inf \left\{\phi_{i}(x): i \in I\right\}, \quad x \in X .
$$

It is easy to see that $\inf \left\{\phi_{i}\right\}$ is concave and nonnegative. We also clearly have

$$
G^{-}\left(\inf \left\{\phi_{i}\right\}\right)=\bigcap_{i} G^{-}\left(\phi_{i}\right)
$$

so that $\inf \left\{\phi_{i}\right\}$ again belongs to $\operatorname{SCNC}(X)$.

Let $Z \subseteq X$ be an arbitrary subset of $X$, and let $f$ be an arbitrary real-valued function on $Z$. Consider the set of $\phi$ in $\operatorname{SCNC}(X)$ such that $\phi$ dominates $f$ on $Z$ (i.e., $\phi(z) \geq f(z)$ for all $z \in Z$ ). Evidently, the infimum of such $\phi$ will again dominate $f$. Thus if there are any elements of $\operatorname{SCNC}(X)$ dominating $f$ on $Z$, there is a minimum one. In particular, given a point $x_{0} \in X$, there is a minimum element of $\operatorname{SCNC}(X)$ taking the value 1 at $x_{0}$.

Proposition 1: Given $x_{0} \in X$, define a function $E_{X}\left(x_{0}, x\right)$ on $X$ by

$$
\begin{aligned}
E_{X}\left(x_{0}, x\right) & =\sup \left\{(t-1) / t: x_{0}+t\left(x-x_{0}\right) \in X\right\}, \quad x \in X, \\
& =\sup \left\{s \in[0,1]: x=s x_{0}+(1-s) z \text { for some } z \in X\right\} .
\end{aligned}
$$

Then $E_{X}\left(x_{0}, \cdot\right)$ is the minimum among elements of $\operatorname{SCNC}(X)$ taking the value 1 at $x_{0}$.

REMARK. In pictorial terms we may describe the (closure of the) graph of $E_{X}\left(x_{0}, \cdot\right)$ as the surface of the cone with base $X \times\{0\}$ and vertex $\left(x_{0}, 1\right)$.

ProOF. In $V \times \mathbf{R}$, let $C\left(X, x_{0}\right)$ denote the closed convex hull of the points $(x, 0)$, $x \in X$, and the point $\left(x_{0}, 1\right)$. Since $X$ is convex, the convex hull of $X \times\{0\}$ and $\left(x_{0}, 1\right)$ is the set $\left\{\left(s x_{0}+(1-s) y, s\right): y \in X, 0 \leq s \leq 1\right\}$ and $C\left(X, x_{0}\right)$ will be the closure of this set. Suppose $x \neq x_{0}$, and

$$
(x, r)=\left(s x_{0}+(1-s) y, s\right) \text {. }
$$

Then $r=s<1$, and

$$
y=x_{0}+(1-s)^{-1}\left(x-x_{0}\right)
$$

belongs to $X$. Setting $t=(1-s)^{-1}$ we have

$$
r=s=1-t^{-1}=(t-1) / t .
$$

From the convexity of $X$ it is clear that if $(x, r)$ is in $C\left(X, x_{0}\right)$, then so is $\left(x, r^{\prime}\right)$ for $0 \leq r^{\prime} \leq r$. Hence $C\left(X, x_{0}\right)$ satisfies conditions (4)(a)(b), and comparing (4)(c)(d) with (6) shows

$$
C\left(X, x_{0}\right)=G^{-}\left(E_{X}\left(x_{0}, \cdot\right)\right)
$$

Furthermore, if $\phi$ is any function in $\operatorname{SCNC}(X)$ such that $\phi\left(x_{0}\right) \geq 1$, then obviously $G^{-}(\phi) \supseteq C\left(X, x_{0}\right)$, whence $\phi(x) \geq E_{X}\left(x_{0}, x\right)$. This proves Proposition 1 . 
Given a point $x_{0}$ in $X$, we say $X$ is conical at $x_{0}$ if there exist

(i) a neighborhood $U$ of $x_{0}$ in $V$ and,

(ii) a closed convex cone $C \subseteq V$, such that

$$
X \cap U=\left(C+x_{0}\right) \cap U .
$$

That is, near $x_{0}$, the set $X$ looks like a translated cone. Note that $C$ need not be a proper, also called pointed, cone. In particular, we could take $C=V$. Thus $X$ is conical at all of its interior points.

PROPOSITION 2. (a) If $E_{X}\left(x_{0}, \cdot\right)$ (cf. formula (6)) is continuous at $x_{0}$, then all functions in $\operatorname{SCNC}(X)$ are continuous at $x_{0}$.

(b) The function $E_{X}\left(x_{0}, \cdot\right)$ is continuous at $x_{0}$ if and only if $X$ is conical at $x_{0}$.

Proof. (a) Suppose $E_{X}\left(x_{0}, \cdot\right)$ is continuous at $x_{0}$. Then given $\varepsilon>0$, there is a neighborhood $U$ of $x_{0}$ such that $E_{X}\left(x_{0}, x\right)>1-\varepsilon$ for $x \in U \cap X$. Consider $\phi \in \operatorname{SCNC}(X)$. By semicontinuity the superlevel set $L^{+}\left(\phi, \phi\left(x_{0}\right)+\varepsilon\right)$ (cf. (2)) is closed, and since it does not contain $x_{0}$, the set $U^{\prime \prime}=V-L^{+}\left(\phi, \phi\left(x_{0}\right)+\varepsilon\right)$ is a neighborhood of $x_{0}$. If $\phi\left(x_{0}\right)=0$, then since $\phi \geq 0$, we see $\left|\phi(x)-\phi\left(x_{0}\right)\right|<\varepsilon$ on $U^{\prime \prime} \cap X$, so $\phi$ is continuous at $x_{0}$. If $\phi\left(x_{0}\right)>0$, then it suffices to show $\phi(x) / \phi\left(x_{0}\right)$ is continuous at $x_{0}$. Hence we may assume $\phi\left(x_{0}\right)=1$. Then on the neighborhood $U \cap U^{\prime \prime} \cap X$ of $x$ in $X$ we have $1+\varepsilon>\phi(x)>E_{X}\left(x_{0}, x\right)>1-\varepsilon$. Hence again $\phi$ is continuous at $x_{0}$.

(b) Let $U$ be an open convex neighborhood of the origin in $V$, with compact closure $\bar{U}$. Then any neighborhood of $x_{0}$ contains a set of the form $x_{0}+\delta U$ for a suitably small number $\delta>0$. Let $\partial U=\bar{U}-U$ be the boundary of $U$. If $C$ is any closed convex cone in $V$ then we have

$$
C=\bigcup_{s \geq 0} s(C \cap \partial U)
$$

Suppose $E_{X}\left(x_{0}, \cdot\right)$ is continuous at $x_{0}$. Then we can find $\delta>0$ such that $E_{X}\left(x_{0}, x\right)>\frac{1}{2}$ for $x \in\left(x_{0}+\delta U\right) \cap X$. Set

$$
B=\left(x_{0}+\delta(\partial U)\right) \cap X, \quad C=\bigcup_{s \geq 0} s\left(B-x_{0}\right) .
$$

Then $C$ is a cone (a union of rays), and clearly

$$
\left(C+x_{0}\right) \cap\left(x_{0}+\delta U\right) \subseteq X \cap\left(x_{0}+\delta U\right) .
$$

For if $x \in C+x_{0}$, then $x=x_{0}+s b, b \in B, s \geq 0$; and if $x \in x_{0}+\delta U$, then $s<1$. Hence $x=(1-s) x_{0}+s\left(x_{0}+b\right) \in X$, since $X$ is convex. I claim that in fact the inclusion (8) is an equality. To verify this, consider a point $v$ in $\left(x_{0}+\delta U\right) \cap X$. Assume $y \neq x_{0}$. For suitable $t \geq 1$ the point $z=x_{0}+t\left(y-x_{0}\right)$ will be in $x_{0}+\delta(\partial U)$. If we show $z \in X$, the claim will be established. Suppose $z \notin X$. Since $X$ is closed and convex, there is a number $a, 0<a<1$ such that the points $z_{r}=x_{0}+r\left(z-x_{0}\right)$ are in $X$ for $r \leq a$, and are not in $X$ for $r>a$. We see then that $E_{X}\left(x_{0}, z_{a}\right)=0$. But since clearly $z_{a} \in X \cap\left(x_{0}+\delta U\right)$, this contradicts our choice of $\delta$. Thus inclusion $(8)$ is an equality, and $X$ is conical at $x_{0}$. 
Conversely, suppose $X$ is conical at $x_{0}$. Let $U$ be a convex neighborhood of the origin, and $C$ a closed convex cone such that

$$
\text { (a) }\left(x_{0}+U\right) \cap X=x_{0}+(C \cap U) \text {. }
$$

Then for $0<a \leq 1$, the set

$$
U_{a}^{\prime}=\left(x_{0}+a U\right) \cap X=x_{0}+a(C \cap U)
$$

will be a neighborhood of $x_{0}$ in $X$. Taking $t$ in formula (6) to be $\frac{1}{a}$ we see that $E_{X}\left(x_{0}, x\right) \geq 1-a$ if $x \in U_{a}^{\prime}$. Hence $E_{X}\left(x_{0}, \cdot\right)$ is continuous at $x_{0}$. This proves Proposition 2 .

The connection of the above two results with automatic continuity is provided by the following result. Given a point $x_{0} \in X$, we say $X$ is polyhedral at $x_{0}$ if there is a polyhedral closed convex subset $P_{x_{0}} \subseteq X$ such that $P_{x_{0}}$ contains a neighborhood of $x_{0}$ in $X$. We say $S$ is locally polyhedral if $X$ is polyhedral at each of its points. We say $X$ is semilocally polyhedral or boundedly polyhedral if any compact subset $C \subseteq X$ is contained in a polyhedral subset $P \subseteq X$.

This definition may seem superficially different from the definition of boundedly polyhedral in [GKR, p. 867], but it is easily seen to be equivalent.

PROPOSITION 3. The following are equivalent:

(i) $X$ is conical at each of its points.

(ii) $X$ is locally polyhedral.

(iii) $X$ is semilocally polyhedral.

(iv) All $\phi \in \operatorname{SCNC}(X)$ are continuous.

REMARKS. (a) The implication (ii) $\Rightarrow$ (i) has a local version: if $X$ is polyhedral at $x_{0}$, then $X$ is conical at $x_{0}$; the implication (i) $\Rightarrow$ (ii) has no such local version.

(b) The implication (i) $\Rightarrow$ (ii) can be deduced from $[\mathbf{K}]$ (see especially Theorems 4.1 and 4.7 ), but we give a short proof.

(c) The equivalence (iii) $\Leftrightarrow$ (iv) amounts more or less to the equivalence $(B P) \Leftrightarrow$ $S$ of Theorem 2 of [GKR].

ProOF. The implication (iii) $\Rightarrow$ (ii) is trivial. The implication (ii) $\Rightarrow$ (i) is routine; we omit its proof. The equivalence (i) $\Leftrightarrow$ (iv) follows from Proposition 2 . The implication (ii) $\Rightarrow$ (iii) is Proposition 2.17 of $[\mathbf{K}]$.

We prove (i) $\Rightarrow$ (ii) by induction on $\operatorname{dim} X=\operatorname{dim} V$. If $\operatorname{dim} V=2$, it is immediate since closed convex cones in 2 -space are polyhedral. It follows directly from the definitions that if $X$ is conical at every point, and $A \subseteq V$ is an affine subspace, then $X \cap A$ is conical at every point. Hence, if $\operatorname{dim} A<\operatorname{dim} V$ we may assume $A \cap X$ is locally polyhedral. If the neighborhood $U$ in the proof of Proposition (2b) (see inclusion (8)) is chosen so that its closure $\bar{U}$ is polyhedral, then (using (ii) $\Rightarrow$ (iii)) we see that the intersection of $X$ with each codimension one face of $x_{0}+\delta \bar{U}$ will be polyhedral. Hence the set $B$ is polyhedral (in the sense that it is a finite union of convex polyhedra; it may not be convex), and in particular has a finite number of extreme points. By (8) (which, we recall, is an equality, not just an inclusion) we see that $X \cap\left(x_{0}+\delta \bar{U}\right)$ is the convex hull of (the extreme points of) $B$ and of $x_{0}$, and so is polyhedral. 


\section{REFERENCES}

[GKR] D. Gale, V. Klee and R. T. Rockafellar, Convex functions on convex polytopes, Proc. Amer. Math. Soc. 19 (1968), 867-873.

[K] V. Klee, Some characterizations of compact polyhedra, Acta Math. 102 (1959), 79-107.

[R] R. T. Rockafellar, Convex analysis, Princeton Univ. Press, Princeton, N.J., 1970.

Department of Mathematics, Yale University, New Haven, Connecticut 06520 